\title{
ABSTRACTS OF CURRENT LITERATURE
}

\section{SCANDINAVIAN JOURNAL OF MEDI- CINE AND SCIENCE IN SPORTS}

\author{
Inflammation and Atrial Remodeling After a Mountain \\ Marathon
}

With the increasing popularity of long-distance sports, endurance athletes are at risk for developing atrial arrhythmias, especially fibrillation. Although the pathophysiology of exercise-induced atrial fibrillation is multifactorial, the authors of this study hypothesize that atrial remodeling and acute inflammation play a key role. The investigators performed a longitudinal study on elite runners at the 2010 Jungfrau Marathon, a popular trail marathon in the Swiss Alps. Male participants 20 to 40 years old who had completed the 2009 Jungfrau Marathon and did not have a personal or family history of cardiac disease were enrolled. Subjects had the following tests performed: electrocardiogram with signal average P-wave duration (SAPWD), transthoracic echocardiography, 24-hour Holter monitor, cardiopulmonary exercise testing, 24-hour blood pressure measurements, and blood laboratory analysis. A combination of these various tests was performed at baseline, immediately after the race, and 1 and 8 days after the race.

A total of 10 participants were enrolled with similar baseline characteristics. Although the left and right atrial volumes remained unchanged, the SAPWD increased significantly directly after the race but returned to baseline during followup. The cardiac biomarkers proatrial natriuretic peptide (proANP) and troponin also increased immediately after the race but decreased to baseline, thought to be caused by a transient increase in atrial stretch and reversible myocyte membrane leak. The C-reactive protein (CRP) increased 1 day after the race but decreased to baseline 8 days later.

By using the SAPWD as a surrogate marker to represent atrial edema and remodeling, the authors proposed a transient and significant atrial conduction delay after the trail marathon. In conclusion, the authors believe their data represent an acute inflammatory process that plays an important role in atrial remodeling and could predispose endurance athletes to atrial fibrillation. Future studies would include structural cardiac imaging, such as cardiac magnetic resonance imaging. Study limitations include small sample size, influence of high altitude, and the use of SAPWD as an indirect measure of myocardial edema.

(Scand J Med Sci Sports. 2012 Dec 18 [Epub ahead of print]) M Wilhelm, T Zueger, S De Marchi, et al.

Prepared by Matthew Stewart, MD, University of Utah Emergency Medicine Resident, Salt Lake City, UT, USA.

\section{PUBLIC LIBRARY OF SCIENCE}

\section{Protective Effect of Tetracycline Against Dermal Toxicity Induced by Jellyfish Venom}

Worldwide there are more than 10,000 reported jellyfish stings annually that can potentially cause local dermatonecrosis and systemic symptoms including cardiovascular instability, respiratory distress, and anaphylaxis. When dermal tissue is exposed to jellyfish venom, there is a significant inflammatory reaction and upregulation of matrix metalloproteinase (MMP) activity. Other than its use as an antibiotic, tetracycline is also an effective metalloproteinase inhibitor but has never been studied as a topical treatment for jellyfish stings. This study investigated the role of MMP activity in dermal toxicity after a sting from the Nemopilema nomurai Scyphozoan jellyfish and the ability of tetracycline to inhibit the clinical sequelae after envenomation.

After treating human keratinocyte and mouse fibroblast models with isolated $N$ nomurai jellyfish venom and different concentrations of tetracycline, the investigators measured MMP activity with gelatin zymography, electrophoresis, and Western blot analysis. Additionally, the investigators performed in vivo experiments using envenomated rabbit models treated with lanolin cream alone or containing tetracycline. Finally, the authors analyzed the histological effects of envenomated rabbit skin after treatment with tetracycline.

After venom exposure, MMP activity significantly increased but was suppressed in the presence of tetracycline. In the in vivo rabbit models, the maximal skin lesion size was noted after 24 hours and steadily decreased over a period of 48 hours. Topical application of lanolin cream with tetracycline to envenomated areas of skin significantly reduced maximal lesion size at 24 hours. Additionally, histological analysis showed a decreased level of hemorrhage and neutrophilic infiltration for skin treated with tetracycline.

This study suggested that tetracycline is able to downregulate MMP activity induced by $N$ nomurai jellyfish venom and can also decrease maximal lesion size when applied topically. The authors conclude that tetracycline creams may be beneficial as a first-aid dermal treatment after jellyfish envenomation. Limitations of the study include applicability to human skin and accessibility to tetracycline cream shortly after jellyfish envenomation.

(PLoS One. 2013;8:e57658) C Kang, YB Jin, J Kwak, et al. Prepared by Matthew Stewart, MD, University of Utah Emergency Medicine Resident, Salt Lake City, UT, USA. 